\title{
The Benefit of Renewable Indonesian Traditional Therapy to Enhance Recovery of Physical-Mental Illness
}

\author{
Sudjiwanati \\ Universitas Wisnuwardhana, Indonesia \\ sudjiwanati@yahoo.com
}

\begin{abstract}
Renewable Indonesian traditional therapies: holomerispot manipulation is a natural, nochemical treatment that can be used to improve mental health and reduce symptoms of physical illness. This study aims to prove the influence of holomerispot manipulation therapy on a key immune mediator: cytokine interleukin-6 (IL-6). The research method used was experimental research with pre and post-therapy repetition with control group. The sample was 20 people divided into 2 groups, which each group got different treatment. Group 1 was the control group and group 2 was the treatment group with holomerispot manipulation and art therapy. The results suggested that the treatment group showed lower IL-6 values compared to the control group. Based on the results of the psychological tests, the treatment group showed a decrease in the level of anxiety and depression, which was better than the control group. The conclusion of this study is that holomerispot manipulation therapy and Art therapy can improve healing and reduce symptoms of mentalphysical illness of the patients. The holomerispot therapy manipulation and art therapy are able to reduce physical-mental illness through the degradation levels of IL-6.
\end{abstract}

Keywords: Holomerispot Manipulation, Art Therapy, Physical-Mental Illness

\section{INTRODUCTION}

Many people experience chronic and recurrence physical illness because of the presence of comorbidities. The most common comorbidities of illness experienced by a person are not only mental illnesses, such as schizophrenia and other diseases related to mental illness, but also physical comorbidities, such as migraine, cephalalgia, bronchial asthma, angina pectoris, gastritis, and pain in the body which are chronic and exacerbated. About $30 \%$ to $45 \%$ of the patients with protracted diseases can experience comorbidities with depressive disorders and patients with comorbidity disease will experience a low quality of life [1].

While many cases of treatment of physical-mental comorbidities are still not able to provide healing, in spite

\author{
Suparno \\ Universitas Wisnuwardhana, Indonesia \\ cipto_parno@yahoo.com
}

of the undergoing various medical treatments, such as chemotherapy and the other therapies. In some cases of the people with comorbidities diseases, they often seek and take alternative methods, such as using herbs and other alternatives medicine in order to reduce symptoms and get healed.

Therefore, it needs some efforts to help improve the recovery rate of a person who suffers comorbid disease by using a natural and non-harmful method to the patient. One of the methods offered is the Double PhysicalMental Therapy which is designed as an added/complementary therapy for modern medication. This can increase the mend of comorbid both physically and mentally.

The dual physical therapy method is a composite of the two therapies which are holomerispot manipulation and art therapy. The manipulation of holomerispot is the development of traditional Indonesian therapy of kerokan (scrape) along with knowledge of acupuncture points. Holomerispot manipulation is a non-invasive stimulation therapy performed by rubbing with fingers at accupointtrigger point-tender point.

Stimulation of acupuncture points can lead to regeneration of several organs (via stem cells) that strongly support the process of preventing or healing various disorders or diseases, especially those that include comorbidities, through meridian and trigger therapies point [2],[3]. According to Joukar:

"Acupuncture stimulates local blood flow and muscle affairs through neurogenic formation of vasoactive mediators and autonomic reflexes. It modulates hypothalamic-pituitary control of the autonomic and neuroendocrine systems, especially microcirculation, response of smooth and striated muscle, and local and general thermregulation" [4].

The stimulation effects of acupuncture points are mediated through some physiological pathways, such as the hypothalamus-pituitary-adrenal (HPA) axis, sympathetic and parasympathetic (cholinergic) pathways, as well as action on histamine, cytokines (such as TNF- $\alpha$, IL-1 $\beta$, IL-6 and IL-10) [5].

Interleukin-6 is a cytokine that is involved in the response to inflammation and infection, regulation of metabolic, regenerative, and neural processes. The basic work of interleukin- 6 is by stimulating the target cell via 
a membrane-bound, interleukin-6 receptor, which upon ligand binding associates with the signaling receptor protein gp130 [6]. Interleukin 6 (IL-6), will soon respond and be produced in the event of infection and tissue injury, IL-6 will work in performing defense functions through stimulation of acute phase response, histopsiesis, and immune reactions. The work process of IL-6 is tightly controlled by a mechanism, such as transcriptional and posttranscriptional. The continuous dysregulation of IL-6 synthesis can provide a pathological effect on cases of chronic inflammation and autoimmunity [7].

Improvement of expected health conditions with holomerispot manipulation therapy is an accretion of the patient's immune system. The parameters that can be used to see the condition of the immune system are IL-6. IL-6 is a cytokine that has a pro and anti-inflammatory properties that regulate the immune system. IL-6 levels for healthy humans range from 0.002-0.006 ng/ml (2-6 $\mathrm{pg} / \mathrm{ml})$. IL-6 levels will rise up when the body is at a state of illness, and also if someone has mental disorders such as release and depression [8],[9].

The Art Therapy in physical-mental therapy is useful to help patients reduce psychiatric disorders caused by chronic illness experienced. The Art Therapy is a way of mental health therapy for clients who encounter trauma, anxiety, depression, chronic illness, and substance abuse by exploring available art material. It could be the art material that is provided by either the therapist or the client. Then, those materials are used to perform the therapeutic process.

The Art Therapy is a therapy that employs art materials so that the patients can express and reflect before the therapist. To have the art therapy, the patient does not need to have previous experience or skills in the art because the goal is not on how to create arts but how the patient can make changes and develop their mental health with the use of art materials. Art therapy can take a role dealing with mental health problems by providing alternative communication media so that the message can be conveyed and understood [10].

Generally, the benefits of The Art Therapy are to improve and maintain mental health, emotional wellbeing, self-expression, communication, awareness, security, crisis, creativity, better stress levels, cope feeling, anxiety, and fear.

\section{METHOD}

The research design used in this research is experiment; the one-group pretest-posttest design is with a control group. The purpose of this study is to prove that it is effective and potential to reduce symptoms or comorbid complaints of physical-mental illness (multiple efficacy for physical symptoms or comorbidities) which also applies to mass therapy. This study is directed to obtain supporting evidence in the form of improved laboratory parameters Biomedical IL-6.

The number of samples used is 20 patients divided into two groups. Each group was treated differently. Group I was a control group and group II was treated with holomerispot manipulation therapy and Art therapy. Data were collected using pre- and post-treatment with blood sampling to see Biomedical laboratory parameters of cytokine IL-6 as measured by the Enzyme Linked Immunosorbent Assay (ELISA) and patients' mental health data were collected by psychological tests using graphical tests.

The treatment group received holomerispot manipulation therapy and art therapy. Holomerispot manipulation therapy was done by rubbing the acupuncture points that have been adapted to the type of disease of the patients. Each participant performed holomerispot manipulation therapy at acupuncture points with a duration of 5 to 10 minutes three times a day for 2 months. The main accupoint used for holomerispot manipulation therapy was ST-36 (Suzanli), and other accupoints used were adjusted to the patient's physicalmental disorders. The type of physical-mental disorders of the patient was obtained from the results of the examination and the patient' health records.

Art therapy was given to patients 4 times within 2 months. Art therapy given to patients in the treatment group was by drawing free and coloring. The results of art therapy were recorded and analyzed by a psychologist to determine the psychological development of the patients.

Laboratory data analysis was performed with statistical tests to see the differences and compare the results between the treatment groups. The statistical test used was $\mathrm{t}$ test to see the result difference between treatment groups. Descriptive analysis was also done to interpret the results of psychological tests.

\section{RESULT}

Table 1. The mean value control treatment group levels of serum IL-6

\begin{tabular}{cccrr}
\hline & & $\mathrm{n}$ & $\begin{array}{c}\text { IL- } \\
\text { 6(ng/ml })\end{array}$ & Sdt. Dev \\
\hline Control & Pre & \multirow{2}{*}{10} & 1.13 & 0.30 \\
& Post & & 1.04 & 0.23 \\
Treatment & Pre & \multirow{2}{*}{10} & 1.10 & 0.37 \\
& Post & & 0.65 & 0.15 \\
\hline
\end{tabular}

Table 1. shows the mean values of laboratory test results from serum IL-6 in the control group and the treatment groups before and after treatment. 
Table 2. Description of the number of patients and the level of anxiety and depression (post therapy)

\begin{tabular}{ccccc}
\hline & \multicolumn{2}{c}{ Control } & \multicolumn{2}{c}{ Treatment } \\
\cline { 2 - 5 } & Anxiety & Depression & Anxiety & Depression \\
\hline Light & 2 & 1 & 4 & 5 \\
Medium & 3 & 5 & 3 & 2 \\
High & 5 & 4 & 2 & 3 \\
\hline
\end{tabular}

Table 2. shows the number of patients with anxiety and depression disorders based on severity after being treated with art therapy.

The result of analysis by using $t$ test and independent sample test method was obtained $t$ value of 4,054>t table $2,5524(\mathrm{p}<0,05)$ which means that the combination treatment of holomerispot manipulation applied to acupoint ST-36 and art therapy had significant influence to decrease value of IL- 6 .

The research, conducted by Hyun-JaJeong et al, observed cytokine changes in chronic headache patients in whom the amount of cytokine IL- 6 before treatment was greater than in the control group. The amount of interleukin (IL)-1 $\beta$, IL-6 and tumor necrosis factor- $\alpha$ $(\mathrm{TNF}-\alpha)$ in LPS culture supernatant significantly increased in the patients with $\mathrm{CH}$ compared to the healthy group $(\mathrm{p}<0,05)$. But those cytokines came down toward the levels of the healthy group $(\mathrm{p}<0,05)$ after treatment with acupuncture, although the level still remained elevating [8]. In some experiments using animal experiments showed that acupuncture therapy using various techniques could decrease the amount of cytokine IL-6 and improve the immune system[11],[12],[13]. Nascimento de Souza et, al also found that acupuncture at ST36 and GV3 is able to promote partial recovery of locomotion, and this improvement could be associated with reduced lesion size and an anti-inflammatory action by modulating the balance of pro and anti -inflammatory cytokines [11]. Acupuncture at ST36 significantly reduced collagen-

disorder and depression average. Data in Table 2. shows the number of patients with lower anxiety and depression disorder in the treatment group than the control group. The treatment group had 4 with low anxiety disorder and 5 with light depression after receiving treatment of art therapy while the control group was fewer. The result of the psychological test was in synergy with the result of the laboratory test which obtained the average data of cytokine IL-6. Post-test in the treatment group had lower value than the control group. Based on the data in Table 1, it can be seen that the average of patients in the treatment group had a lower cytokine IL-6 (post-test) score of $0.65 \pm 0.15$ compared with the control group of $1.04+0.23$.

induced arthritic, IL-6, TNF- $\alpha$, INF- $\gamma$, collagen antibody, $\operatorname{IgG}$ and $\operatorname{IgM}$ levels and prevented knee joint destruction [14].

Psychological test results also showed that in the treatment group, patients got better condition than the control group after getting art therapy the level of anxiety
The results of this research indicated that the physical-mental conditions are essentially related to each other and may affect the amount of cytokine IL- 6 in the blood. The condition of a person with a mental disorder such as major depressive disorder may increase the amount of cytokine IL- 6 because it can affect to proinflammatory cytokines [15]-[17].

This research still cannot distinguish the improvement of patients' mental condition influenced by art therapy and how strong art therapy affects the improvement of mental condition of patients. But in this research, it can be seen that a combination of holomerispot manipulation therapy and art therapy resulted in improved physical-mental conditions in patients by decreasing levels of cytokine IL-6 in the blood.

\section{CONCLUSION}

Based on the results of the research, it can be concluded that the holomerispot manipulation therapy and art therapy were given to increase the recovery of physical-mental comorbid disease in the treatment group. Improved recovery was demonstrated by an improvement in the value of cytokine IL-6 laboratory parameters after treatment received a lower value than before treatment compared with the control group.

\section{REFERENCES}

[1] Z. Yang et al., "The effectiveness of acupuncture for chronic pain with depression A systematic review protocol," Medicine (Baltimore)., vol. 96, no. October, pp. 47-8800, 2017.

[2] P. T. Dorsher and P. M. McIntosh, "Acupuncture's effects in treating the sequelae of acute and chronic spinal cord injuries: A review of allopathic and traditional Chinese medicine literature," Evidence-based Complement. Altern. Med., vol. 2011, 2011.

[3] L. Johnston, Alternative Medicine and Spinal Cord Injury. New York: Demos Medical Publishing, Inc, 2006.

[4] M. Joukar, "Influence of Acupuncture on The Inflammatory Processes and Immunity," South Baylo University, 2016.

[5] Z. Panagiotis, G. Vasileios, V. Anastasios, S. Konstantinos, V. Georgios, and T. Stamatios, "Acupuncture Treatment for Allergic Rhinitis: Why should Physicians Recommend it?," J. Clin. Exp. Neuroimmunol., vol. 2, no. 1, pp. 2-5, 2017.

[6] J. Scheller, A. Chalaris, D. Schmidt-Arras, and S. Rose-John, "The pro- and anti-inflammatory properties of the cytokine interleukin-6," Biochim. 
Biophys. Acta - Mol. Cell Res., vol. 1813, no. 5, pp. 878-888, 2011.

[7] T. Tanaka, M. Narazaki, and T. Kishimoto, "Il-6 in inflammation, Immunity, And disease," Cold Spring Harb. Perspect. Biol., vol. 6, no. 10, pp. 116, 2014.

[8] J. Cohen, "Inhibiting Interleukin-6 (IL-6): The Key To Health, Successful Aging and Vitality," 2018. [Online]. Available: https://selfhacked.com/blog/interleukin-6/.

[9] A. O’Donovan et al., "Clinical anxiety, cortisol and interleukin-6: Evidence for specificity in emotion-biology relationships," Brain Behav Immun, vol. 24, no. 7, pp. 1074-1077, 2010.

[10] D. Edwards, Art Therapy, 1st ed. London: Sage Publications, 2004.

[11] R. Nascimento de Souza, F. K. Silva, and M. Alves de Medeiros, "Bee Venom Acupuncture Reduces Interleukin-6, Increases Interleukin-10, and Induces Locomotor Recovery in a Model of Spinal Cord Compression," JAMS J. Acupunct. Meridian Stud., vol. 10, no. 3, pp. 204-210, 2017.

[12] M. V. R. Scognamillo-Szabó, G. H. Bechara, and F. de Q. Cunha, "Effect of acupuncture on TNF- $\alpha$, IL-1 $\beta$ and IL-10 concentrations in the peritoneal exudates of carrageenan-induced peritonitis in rats," Ciência Rural, vol. 35, no. 1, pp. 103-108, 2005.
[13] M. H. Cha, T. S. Nam, Y. Kwak, H. Lee, and B. H. Lee, "Changes in cytokine expression after electroacupuncture in neuropathic rats," Evidencebased Complement. Altern. Med., vol. 2012, 2012

[14] Y. K. Yim et al., "Electro-acupuncture at acupoint ST36 reduces inflammation and regulates immune activity in collagen-induced arthritic mice,' Evidence-based Complement. Altern. Med., vol. 4, no. 1, pp. 51-57, 2007.

[15] H. C. Miranda et al., "Higher than Normal Plasma Iinterleukin-6 Concentrations in Brazilian Patients with Mood Disorders," Braz. Arch. Biol. Technol., vol. 54, no. August, pp. 717-722, 2011.

[16] U. Faghihi, S. Patel, S. Bake, and M. A. Hook, "Depression-Like Signs in a Rat Model of Spinal Cord," pp. 176-195, 2017.

[17] J. L. McDonald, A. W. Cripps, P. K. Smith, C. A. Smith, C. C. Xue, and B. Golianu, "The antiinflammatory effects of acupuncture and their relevance to allergic rhinitis: a narrative review and proposed model.," Evid. Based. Complement. Alternat. Med., vol. 2013, p. 591796, 2013. 